\title{
Dynamic contrast-enhanced magnetic resonance imaging perfusion characteristics in meningiomas treated with resection and adjuvant radiosurgery
}

\author{
Swathi Chidambaram, MD, ${ }^{1}$ Susan C. Pannullo, MD, ${ }^{1}$ Michelle Roytman, MD, ${ }^{2}$ \\ David J. Pisapia, MD, ${ }^{3}$ Benjamin Liechty, MD, ${ }^{3}$ Rajiv S. Magge, MD, ${ }^{4}$ Rohan Ramakrishna, MD, ${ }^{1}$ \\ Philip E. Stieg, PhD, MD, ${ }^{1}$ Theodore H. Schwartz, MD, ${ }^{1}$ and Jana Ivanidze, MD, PhD² \\ Departments of ${ }^{1}$ Neurological Surgery; ${ }^{2}$ Radiology, Division of Neuroradiology, Division of Molecular Imaging and Therapeutics; \\ and ${ }^{3}$ Pathology and Laboratory Medicine; and ${ }^{4}$ Weill Cornell Medicine, Cornell University, New York, New York
}

\begin{abstract}
OBJECTIVE There is a need for advanced imaging biomarkers to improve radiation treatment planning and response assessment. T1-weighted dynamic contrast-enhanced perfusion MRI (DCE MRI) allows quantitative assessment of tissue perfusion and blood-brain barrier dysfunction and has entered clinical practice in the management of primary and secondary brain neoplasms. The authors sought to retrospectively investigate DCE MRI parameters in meningiomas treated with resection and adjuvant radiation therapy using volumetric segmentation.

METHODS A retrospective review of more than 300 patients with meningiomas resected between January 2015 and December 2018 identified 14 eligible patients with 18 meningiomas who underwent resection and adjuvant radiotherapy. Patients were excluded if they did not undergo adjuvant radiation therapy or DCE MRI. Demographic and clinical characteristics were obtained and compared to DCE perfusion metrics, including mean plasma volume $\left(v_{p}\right)$, extracellular volume $\left(v_{e}\right)$, volume transfer constant $\left(K^{\text {trans }}\right)$, rate constant $\left(k_{e p}\right)$, and wash-in rate of contrast into the tissue, which were derived from volumetric analysis of the enhancing volumes of interest.
\end{abstract}

RESULTS The mean patient age was 64 years (range $49-86$ years), and $50 \%$ of patients (7/14) were female. The average tumor volume was $8.07 \mathrm{~cm}^{3}$ (range $0.21-27.89 \mathrm{~cm}^{3}$ ). The median Ki-67 in the cohort was $15 \%$. When stratified by median $\mathrm{Ki}-67$, patients with $\mathrm{Ki}-67$ greater than $15 \%$ had lower median $v_{p}(0.02$ vs $0.10, p=0.002)$, and lower median wash-in rate (1.27 vs $4.08 \mathrm{sec}^{-1}, \mathrm{p}=0.04$ ) than patients with $\mathrm{Ki}-67$ of $15 \%$ or below. Logistic regression analysis demonstrated a statistically significant, moderate positive correlation between $v_{e}$ and time to progression $(r=0.49, p<0.05)$. Furthermore, there was a moderate positive correlation between $K^{\text {trans }}$ and time to progression, which approached, but did not reach, statistical significance $(r=0.48, p=0.05)$.

CONCLUSIONS This study demonstrates a potential role for DCE MRI in the preoperative characterization and stratification of meningiomas, laying the foundation for future prospective studies incorporating DCE as a biomarker in meningioma diagnosis and treatment planning.

https://thejns.org/doi/abs/10.3171/2019.3.FOCUS1954

KEYWORDS DCE MRI; meningioma; radiosurgery; perfusion

$\mathrm{M}$ ENINGIOMAS are the most common primary intracranial tumors. Meningiomas are typically treated with surgery and adjuvant radiation in cases of subtotal resection and/or higher histopathological grade. ${ }^{14,16}$ Postoperative MRI appearance is the gold standard for adjuvant treatment planning, specifically stereotactic radiosurgery (SRS). However, MRI can have limited sensitivity and specificity in cases demonstrating an infil- trative pattern of growth, osseous or parenchymal invasion, and/or postsurgical or postradiation change. Furthermore, a wide variety of intracranial lesions, both benign and malignant, have an MRI appearance that can closely mimic meningioma. ${ }^{17}$ Moreover, conventional MRI does not reliably differentiate between subtypes of meningioma. Thus, there is a need for advanced imaging biomarkers to improve radiation treatment planning and response assess-

ABBREVIATIONS BED = biologically effective dose; $\mathrm{DCE}=$ dynamic contrast-enhanced; $k_{\mathrm{ep}}=$ rate constant; $K^{\text {trans }}=$ volume transfer constant; $r$ CBV = relative cerebral blood volume; SRS = stereotactic radiosurgery; TTP = time to progression; $v_{e}=$ extracellular volume; $v_{p}=$ mean plasma volume.

SUBMITTED January 31, 2019. ACCEPTED March 25, 2019.

INCLUDE WHEN CITING DOI: 10.3171/2019.3.FOCUS1954. 
ment. T1-weighted dynamic contrast-enhanced (DCE) perfusion MRI (DCE MRI) allows quantitative assessment of tissue perfusion and blood-brain barrier dysfunction, and has entered clinical practice in primary and secondary brain neoplasms. ${ }^{7}$ In meningioma, DCE MRI has been proposed as a tool to distinguish between lower- and higher-grade tumors and has also been utilized to differentiate meningiomas from dural-based metastases. . $^{12,19,20}$ Moreover, peritumoral edema surrounding atypical meningiomas has been shown to have distinct perfusion characteristics compared with benign meningiomas. ${ }^{20}$ Based on this prior work, we sought to retrospectively investigate DCE MRI parameters in meningiomas treated with resection and adjuvant radiation therapy and hypothesized that DCE perfusion MRI may be an informative biomarker in radiation therapy response assessment.

\section{Methods \\ Patients}

In this institutional review board approved-, Health Insurance Portability and Accountability Act compliant-retrospective study, patients eligible for analysis were selected using retrospective chart review applying the following selection criteria. Based on a database of pathology-proven resected meningiomas, more than 300 patients who underwent resection between January 2015 and December 2018 were screened for eligibility. Patients were excluded if they did not undergo adjuvant radiation therapy and did not undergo DCE MRI, resulting in 14 eligible patients with 18 meningiomas. In all patients, a histopathological and molecular diagnosis was confirmed by experienced, board-certified neuropathologists.

Demographic and clinical characteristics were obtained from clinical chart review, including tumor location (skull base vs other location), Simpson grade, WHO grade, Ki-67 labeling index, radiation therapy type and dose, and time to progression (TTP). The biologically effective dose (BED) of radiation was calculated using an online calculator (https://www.mdcalc.com/radiation-biologically-effective-dose-bed-calculator) (Table 1). ${ }^{18}$

\section{Imaging}

All patients underwent MRI of the brain on 1.5- or 3-Tesla clinical scanners (Skyra, Aera, Biograph mMR, Siemens Healthcare; Discovery 750w, Signa HDxt, GE Healthcare), which included axial T1-weighted (TR/TE 550-700 $\mu \mathrm{sec} / 7-10 \mu \mathrm{sec}$, slice thickness 3-5 mm) or 3D T1-weighted SPACE (TR/TE 600-700 $\mu \mathrm{sec} / 11-19 \mu \mathrm{sec}$, $120^{\circ}$ flip, slice thickness $1 \mathrm{~mm}$ ), axial T2-weighted (TR/ TE 3200-4000 usec/93-98 usec, slice thickness $5 \mathrm{~mm}$ ), and axial T2-weighted FLAIR or 3D T2-weighted FLAIR (TR/TE 6300-8500 $\mu \mathrm{sec} / 394-446 \mu \mathrm{sec}, 120^{\circ}$ flip angle, slice thickness $1 \mathrm{~mm}$ ). T1-weighted DCE MRI was performed and available for analysis in all of the cases (TR $4 \mu \mathrm{sec}, \mathrm{TE} 1-2 \mu \mathrm{sec}$, flip angle $13^{\circ}$, slice thickness $3 \mathrm{~mm}$, 44 slices to cover the entire lesion volume, 24 phases with 4 phases before and 20 phases after intravenous bolus administration of $0.1 \mathrm{~mL} / \mathrm{kg}$ gadopentetate).

\section{DCE Perfusion Analysis}

Olea Medical 3.0 software was utilized for DCE MRI processing and analysis (Figs. 1 and 2). Analysis was performed on volumes of interest that included the entire enhancing tumor volume, inclusive of all slices (Fig. 1). DCE perfusion metrics, including mean plasma volume $\left(v_{p}\right)$, extracellular volume $\left(v_{e}\right)$, volume transfer constant $\left(K^{\text {trans }}\right)$, rate constant $\left(k_{e p}\right)$, and wash-in rate of contrast into the tissue were derived from volumetric analysis of the enhancing volumes of interest. ${ }^{3}$

\section{Statistical Analysis}

Statistical analysis was performed utilizing GraphPad Prism version 7. Patients were stratified by Simpson grade, pathological WHO grade, Ki-67, tumor location, and SRS dosing strategy, and the Mann-Whitney U-test was performed to identify statistically significant differences between DCE parameters. ANOVA was performed to determine statistical differences when stratification into 3 subgroups was needed (WHO grade, SRS dosing strategy). Linear regression and Spearman correlation were also used to investigate the differences in permeability parameters when compared with TTP. The plots were made using ggplot2 in RStudio version 1.1.463 with $\mathrm{R}$ version 3.5.2 (r-project.org; Fig. 3).

\section{Results}

Clinical and demographic characteristics of the study population and DCE parameters are outlined in Table 1 . A total of 18 tumors in 14 patients were evaluated. Mean patient age was 64 years (range 49-86 years), and 50\% of patients (7/14) were female. The average tumor volume was $8.07 \mathrm{~cm}^{3}$ (range $0.21-27.89 \mathrm{~cm}^{3}$ ).

When stratifying by Simpson grade (I, II, and III vs IV and $\mathrm{V}$ ), there was a trend toward higher median $k_{e p}$, $v_{p}$, and $K^{\text {trans }}$ in the higher Simpson grade group; however, this did not reach statistical significance (Table 2). When stratifying by location, there was a trend toward higher median wash-in rate in skull base tumors. When stratifying by SRS dosing into fractionated versus hypofractionated tumors (with the latter group including tumors treated with other types of radiation therapy), differences in median $k_{e p}, K^{\text {trans }}, v_{e}, v_{p}$, and wash-in rate values did not reach statistical significance, although there was a trend for higher $v_{p}$ in the hypofractionated group compared with the fractionated group (0.03 vs $0.09, \mathrm{p}=0.35$; Table 2). When stratifying into 3 subgroups, with non-SRS radiotherapy (such as brachytherapy and proton-beam radiation therapy), the $\mathrm{p}$ value for $v_{p}$ decreased; however, it did not reach statistical significance $(\mathrm{p}=0.06)($ Table 3$)$.

The median Ki-67 in the cohort was $15 \%$. When stratifying by median $\mathrm{Ki}-67$, patients with $\mathrm{Ki}-67$ greater than $15 \%$ had lower median $v_{p}(0.02$ vs $0.10, \mathrm{p}=0.002)$, and a lower median wash-in rate (1.27 vs $\left.4.08 \mathrm{sec}^{-1}, \mathrm{p}=0.04\right)$ than patients with $\mathrm{Ki}-67$ of $15 \%$ or below. The median Ki67 was $15 \%$ for this cohort. Logistic regression analysis demonstrated a statistically significant, moderate positive correlation between $v_{e}$ and TTP $(\mathrm{r}=0.49, \mathrm{p}<0.05)$. There was a moderate positive correlation between $K^{\text {trans }}$ and TTP, which approached, but did not reach, statistical significance $(r=0.48, p=0.05$; Fig. 3). Furthermore, the regression analysis demonstrated significant correlation 


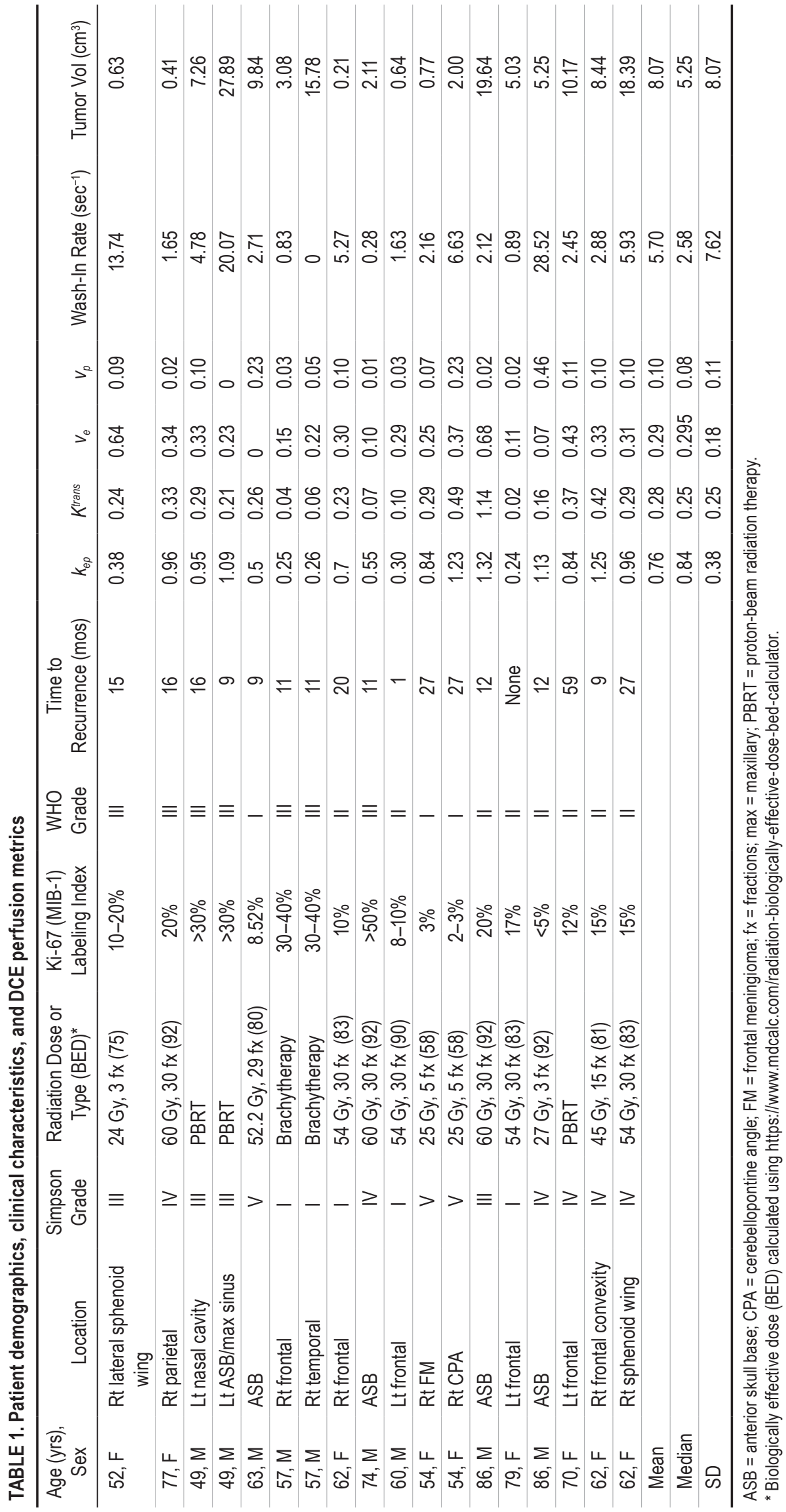



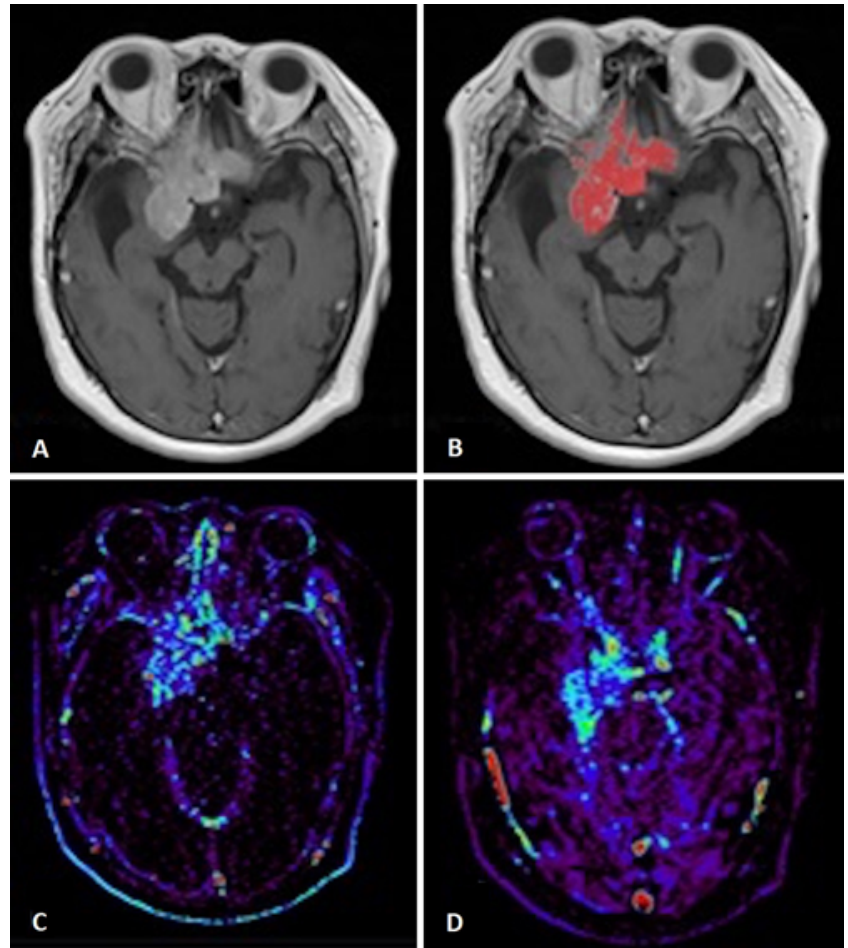

FIG. 1. Representative posttreatment DCE MR images in a 62-year-old woman with recurrent WHO grade II meningioma of the right sphenoid wing with prior craniotomy and fractionated radiation therapy. Axial T1weighted postcontrast image $(\mathrm{A})$ and overlaid semiautomatic volume of interest (B), $K^{\text {trans }} \operatorname{map}(\mathbf{C})$, and $v_{p}$ map (D).

irrespective of WHO grade (Fig. 3). The remaining DCE parameters did not demonstrate statistically significant correlation with TTP.

\section{Discussion}

In this study, we sought to characterize DCE MRI parameters in meningiomas treated with resection and adjuvant radiation therapy. We analyzed clinical and treatment-related information including Simpson grade, tumor location, pathological findings, including $\mathrm{Ki}-67$ index, radiotherapy dosing data, and time to progression of disease. Our findings highlight a potential role for DCE MRI in the preoperative characterization and stratification of meningiomas and lay the foundation for a prospective study incorporating DCE as a biomarker in meningioma diagnosis and treatment planning. Importantly, time to progression after radiation therapy demonstrated a moderate positive correlation, which was statistically significant in the case of $v_{e}$ and approached statistical significance in the case of $K^{\text {trans }}$, indicating that these parameters may be helpful in predicting progression in meningiomas on posttreatment surveillance imaging.

Previous work suggested that DCE MRI may facilitate noninvasive preoperative predictions of intracranial tumor characteristics and may have a potential to allow prognostic decisions and to guide therapies. ${ }^{1,8}$ DCE MRI has also been studied for its potential utility in differentiating tumors by type and grade, as the spatial properties and
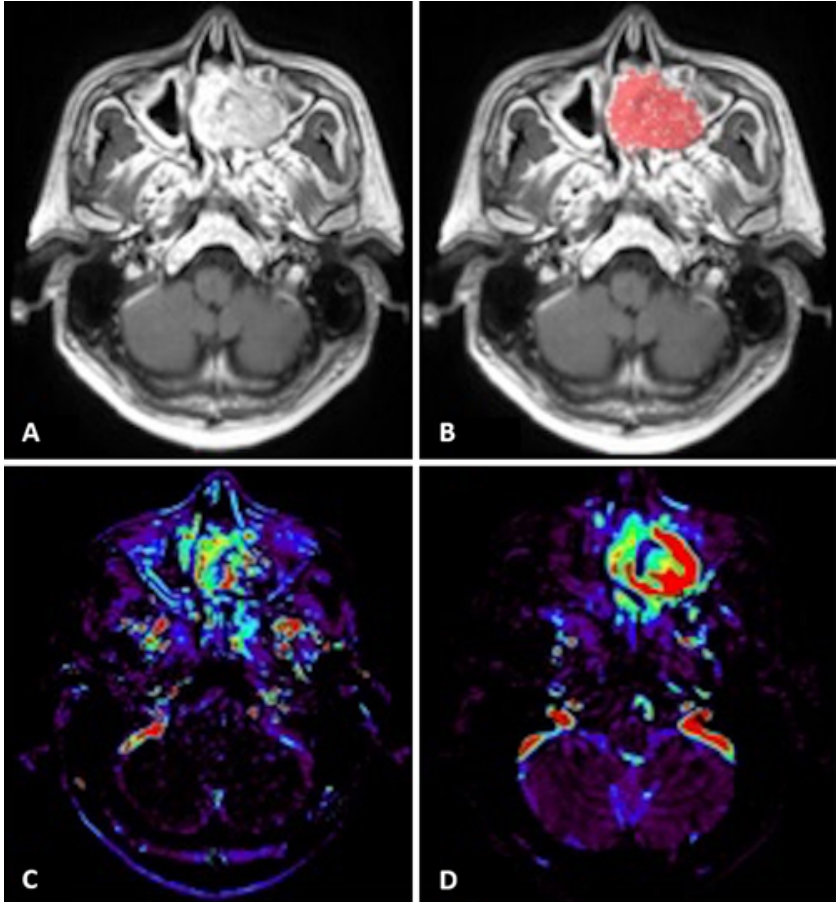

FIG. 2. Representative posttreatment DCE MR images in a 49-year-old man with multiply recurrent WHO grade III meningioma centered in the left maxillary sinus with multiple prior resections and multiple courses of radiation therapy. Axial T1-weighted postcontrast image $(\mathbf{A})$ and overlaid semiautomatic volume of interest (B), $K^{\text {trans }}$ map (C), and $v_{p}$ map (D).

function of the tumor vasculature differ in these circumstances. ${ }^{4,11,13,15}$ In mixed cohorts of intracranial tumors that include meningiomas, gliomas, and metastases, it has been shown that differences in vessel permeability, as captured by perfusion MRI, can help differentiate between meningiomas and intraaxial tumors. ${ }^{2,10,11,13}$

Several studies have previously investigated the potential role of DCE MRI in the characterization of meningiomas. Thus, $K^{\text {trans }}$ was found to have utility in distinguishing atypical meningiomas from typical meningiomas on preoperative DCE MRI..$^{19}$ DCE MRI was also proposed as a biomarker in the diagnosis of rare meningioma variants such as lipomatous variant of metaplastic meningioma and a microcystic meningioma. ${ }^{6}$ Dynamic susceptibility contrastMRI-derived relative cerebral blood volume (rCBV) has been proposed as a biomarker for meningiomas, given data suggesting that rCBV correlated with vascular endothelial growth factor (VEGF) expression and tumor grade. ${ }^{5}$ A recent study suggested that an enhancing cystic lesion with a normalized rCBV greater than $10.3 \mathrm{~cm}^{3}$ or a $K^{\text {trans }}$ greater than $0.88 \mathrm{~min}^{-1}$ should prompt radiologists and surgeons to consider the diagnosis of the rare microcystic meningioma rather than traditional WHO grade I meningioma or high-grade glioma in surgical treatment planning. However, not all data confirm the predictive utility of DCE MRI, which remains an area of active research. ${ }^{9}$

We present here the results from a unique cohort of patients with meningiomas who underwent resection, adjuvant radiotherapy, and DCE MRI and validate the potential utility of DCE MRI in this clinical context. Limitations of 

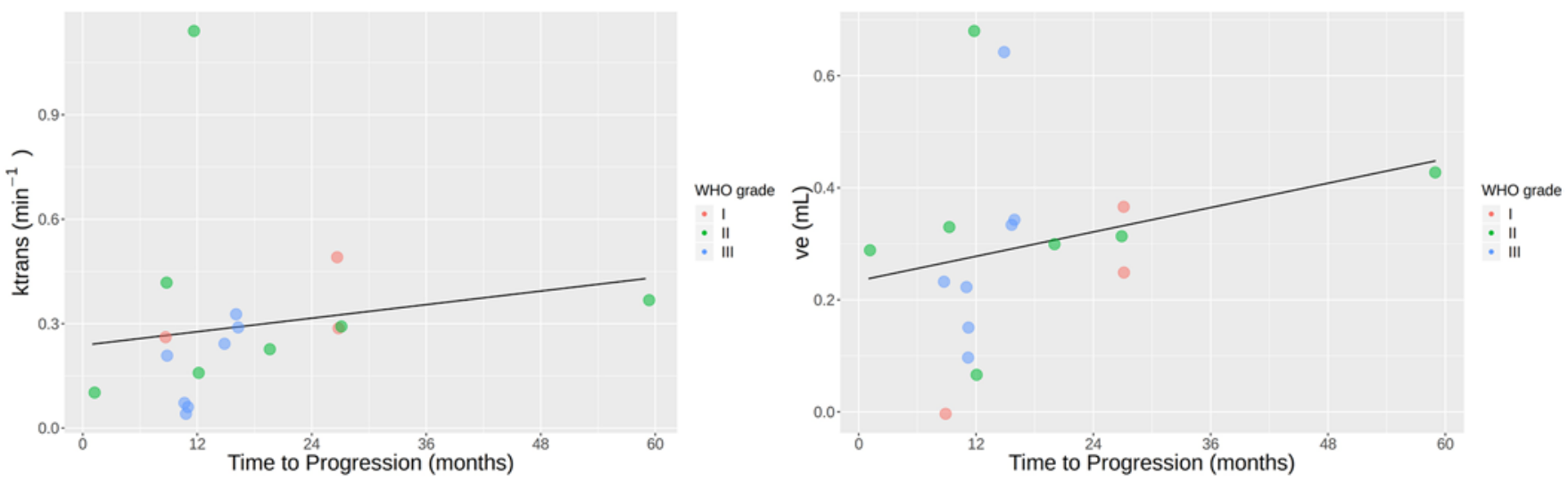

FIG. 3. DCE parameters in meningiomas treated with resection and adjuvant radiosurgery: correlation with TTP. Left: Logistic regression analysis of $K^{\text {trans }}$ and TTP. B: Logistic regression of $v_{e}$ and TTP. Each data point represents a tumor; color indicates WHO grade.

this study include the small sample size and retrospective methodology. Furthermore, the median Ki-67 of this cohort was $15 \%$, indicating an inherent bias toward more aggressive meningiomas. This also implies that DCE MRI was done on more aggressive tumors, likely as a problemsolving tool in treatment planning. Future directions include validating our findings in a prospective cohort including pre- and posttreatment surveillance DCE MRI, allowing for the continued development of DCE MRI as a biomarker of diagnosis and treatment response assessment of meningioma, thereby improving patient outcomes.

\section{Conclusions}

In this study, we aimed to characterize DCE MRI pa- rameters in meningiomas treated with resection and adjuvant radiation therapy. This is the first study of a cohort of patients with meningiomas who have undergone resection and adjuvant radiotherapy that aims to validate the potential usefulness of DCE MRI in this clinical context. Importantly, despite the small sample size, time to progression after radiation therapy demonstrated a moderate positive, statistically significant correlation with $v_{e}$ and a moderate positive correlation with $K^{\text {trans }}$ that approached statistical significance, indicating that these parameters may be helpful in predicting progression in meningiomas on posttreatment surveillance imaging. Furthermore, when stratified by Ki-67, tumors demonstrated statistically significant differences in $v_{p}$ and wash-in rate, which prompts the potential utility of $v_{p}$ and wash-in rate as diagnostic markers

TABLE 2. DCE MRI parameters stratified by Simpson grade, tumor location, Ki-67, and radiotherapy dosing strategy

\begin{tabular}{|c|c|c|c|c|c|c|}
\hline & \multirow[b]{2}{*}{ No. of Meningiomas } & \multicolumn{5}{|c|}{ DCE MRI Parameter* } \\
\hline & & $k_{e p}$ & $K^{\text {trans }}$ & $v_{e}$ & $v_{p}$ & Wash-In Rate $\left(\mathrm{sec}^{-1}\right)$ \\
\hline \multicolumn{7}{|l|}{ Simpson grade } \\
\hline I, II, or III & 9 & 0.38 & 0.21 & 0.29 & 0.03 & 2.12 \\
\hline IV or V & 9 & 0.96 & 0.29 & 0.31 & 0.1 & 2.71 \\
\hline$p$ value & & 0.09 & 0.08 & 0.81 & 0.07 & 0.55 \\
\hline \multicolumn{7}{|l|}{ Tumor location } \\
\hline Skull base & 9 & 0.96 & 0.26 & 0.25 & 0.09 & 5.93 \\
\hline Not skull base & 9 & 0.7 & 0.23 & 0.3 & 0.05 & 1.65 \\
\hline$p$ value & & 0.13 & 0.44 & 0.78 & 0.75 & 0.06 \\
\hline \multicolumn{7}{|l|}{ Ki-67 } \\
\hline$\leq 15 \%$ & 10 & 0.84 & 0.28 & 0.31 & 0.1 & 4.08 \\
\hline$>15 \%$ & 8 & 0.75 & 0.14 & 0.23 & 0.02 & 1.27 \\
\hline$p$ value & & 0.54 & 0.2 & 0.6 & 0.002 & 0.04 \\
\hline \multicolumn{7}{|l|}{ SRS fractionation } \\
\hline Fractionated & 9 & 0.7 & 0.26 & 0.3 & 0.03 & 2.12 \\
\hline Hypofractionated/other & 9 & 0.84 & 0.24 & 0.25 & 0.09 & 4.78 \\
\hline $\mathrm{p}$ value & & 0.98 & 0.86 & 0.81 & 0.35 & 0.26 \\
\hline
\end{tabular}

The Mann-Whitney U-test was performed to determine statistical significance.

* Parameter values are medians. 
TABLE 3. DCE parameters stratified by radiotherapy dosing strategy

\begin{tabular}{lcccc}
\hline & \multicolumn{3}{c}{ Dosing Strategy } & \\
\cline { 2 - 4 } $\begin{array}{c}\text { DCE MRI } \\
\text { Parameter }\end{array}$ & $\begin{array}{c}\text { Fractionated } \\
(\mathrm{n}=9)\end{array}$ & $\begin{array}{c}\text { Hypofractionated } \\
(\mathrm{n}=4)\end{array}$ & $\begin{array}{c}\text { Other } \\
(\mathrm{n}=5)\end{array}$ & $\begin{array}{c}\mathrm{p} \\
\text { Value }\end{array}$ \\
\hline$k_{\mathrm{ep}}$ & 0.70 & 0.84 & 0.99 & 0.71 \\
\hline$K^{\text {trans }}$ & 0.26 & 0.21 & 0.31 & 0.70 \\
\hline$V_{p}$ & 0.03 & 0.05 & 0.17 & 0.06 \\
\hline$V_{e}$ & 0.30 & 0.23 & 0.40 & 0.86 \\
\hline Wash-in rate $\left(\mathrm{sec}^{-1}\right)$ & 2.12 & 2.45 & 10.19 & 0.08 \\
\hline
\end{tabular}

ANOVA was performed to determine statistical significance.

in preoperative treatment planning. Our findings indicate a potential role for DCE MRI in the preoperative characterization and stratification of meningiomas and lay the foundation for a prospective study incorporating DCE as a biomarker in meningioma diagnosis and treatment planning.

\section{References}

1. Bazyar S, Ramalho J, Eldeniz C, An H, Lee YZ: Comparison of cerebral blood volume and plasma volume in untreated intracranial tumors. PLoS One 11:e0161807, 2016

2. Cha S, Yang L, Johnson G, Lai A, Chen MH, Tihan T, et al: Comparison of microvascular permeability measurements, $\mathrm{K}$ (trans), determined with conventional steady-state T1weighted and first-pass $\mathrm{T} 2 *$-weighted MR imaging methods in gliomas and meningiomas. AJNR Am J Neuroradiol 27:409-417, 2006

3. Cuenod CA, Balvay D: Perfusion and vascular permeability: basic concepts and measurement in DCE-CT and DCE-MRI. Diagn Interv Imaging 94:1187-1204, 2013

4. Fujii K, Fujita N, Hirabuki N, Hashimoto T, Miura T, Kozuka T: Neuromas and meningiomas: evaluation of early enhancement with dynamic MR imaging. AJNR Am J Neuroradiol 13:1215-1220, 1992

5. Ginat DT, Mangla R, Yeaney G, Schaefer PW, Wang H: Correlation between dynamic contrast-enhanced perfusion MRI relative cerebral blood volume and vascular endothelial growth factor expression in meningiomas. Acad Radiol 19:986-990, 2012

6. Grand S, Pasquier BM, Hoffmann DM, Krainik A, Ashraf A, Tropres IM, et al: Perfusion MR imaging and $1 \mathrm{H}$ spectroscopy: their role in the diagnosis of microcystic and lipomatous meningiomas. J Neuroradiol 37:185-188, 2010

7. Ivanidze J, Lum M, Pisapia D, Magge R, Ramakrishna R, Kovanlikaya I, et al: MRI features associated with TERT promoter mutation status in glioblastoma. J Neuroimaging [epub ahead of print], 2019

8. Jensen RL, Mumert ML, Gillespie DL, Kinney AY, Schabel MC, Salzman KL: Preoperative dynamic contrast-enhanced MRI correlates with molecular markers of hypoxia and vascularity in specific areas of intratumoral microenvironment and is predictive of patient outcome. Neuro Oncol 16:280291, 2014

9. Keil VC, Pintea B, Gielen GH, Hittatiya K, Datsi A, Simon $\mathrm{M}$, et al: Meningioma assessment: kinetic parameters in dynamic contrast-enhanced MRI appear independent from microvascular anatomy and VEGF expression. J Neuroradiol 45:242-248, 2018

10. Lehmann P, Vallée JN, Saliou G, Monet P, Bruniau A, Fichten A, et al: Dynamic contrast-enhanced T2*-weighted MR imaging: a peritumoral brain oedema study. J Neuroradiol 36:88-92, 2009
11. Lüdemann L, Grieger W, Wurm R, Wust P, Zimmer C: Quantitative measurement of leakage volume and permeability in gliomas, meningiomas and brain metastases with dynamic contrast-enhanced MRI. Magn Reson Imaging 23:833-841, 2005

12. Lui YW, Malhotra A, Farinhas JM, Dasari SB, Weidenheim $\mathrm{K}$, Freeman K, et al: Dynamic perfusion MRI characteristics of dural metastases and meningiomas: a pilot study characterizing the first-pass wash-in phase beyond relative cerebral blood volume. AJR Am J Roentgenol 196:886-890, 2011

13. Nguyen TB, Cron GO, Bezzina K, Perdrizet K, Torres CH, Chakraborty S, et al: Correlation of tumor immunohistochemistry with dynamic contrast-enhanced and DSC-MRI parameters in patients with gliomas. AJNR Am J Neuroradiol 37: 2217-2223, 2016

14. Ostrom QT, Gittleman H, Liao P, Vecchione-Koval T, Wolinsky Y, Kruchko C, et al: CBTRUS Statistical Report: Primary brain and other central nervous system tumors diagnosed in the United States in 2010-2014. Neuro Oncol 19 (suppl_5):v1-v88, 2017

15. Roberts HC, Roberts TP, Brasch RC, Dillon WP: Quantitative measurement of microvascular permeability in human brain tumors achieved using dynamic contrast-enhanced MR imaging: correlation with histologic grade. AJNR Am J Neuroradiol 21:891-899, 2000

16. Rogers L, Barani I, Chamberlain M, Kaley TJ, McDermott M, Raizer J, et al: Meningiomas: knowledge base, treatment outcomes, and uncertainties. A RANO review. J Neurosurg 122:4-23, 2015

17. Starr CJ, Cha S: Meningioma mimics: five key imaging features to differentiate them from meningiomas. Clin Radiol 72:722-728, 2017

18. Vernimmen FJ, Slabbert JP: Assessment of the alpha/ beta ratios for arteriovenous malformations, meningiomas, acoustic neuromas, and the optic chiasma. Int J Radiat Biol 86:486-498, 2010

19. Yang S, Law M, Zagzag D, Wu HH, Cha S, Golfinos JG, et al: Dynamic contrast-enhanced perfusion MR imaging measurements of endothelial permeability: differentiation between atypical and typical meningiomas. AJNR Am J Neuroradiol 24:1554-1559, 2003

20. Zhang H, Rödiger LA, Shen T, Miao J, Oudkerk M: Perfusion MR imaging for differentiation of benign and malignant meningiomas. Neuroradiology 50:525-530, 2008

\section{Disclosures}

The authors report no conflict of interest concerning the materials or methods used in this study or the findings specified in this paper.

\section{Author Contributions}

Conception and design: Ivanidze, Chidambaram, Roytman. Acquisition of data: Ivanidze, Chidambaram, Pannullo, Pisapia, Liechty, Magge, Ramakrishna, Stieg, Schwartz. Analysis and interpretation of data: Ivanidze, Chidambaram, Pisapia, Liechty. Drafting the article: Ivanidze, Chidambaram, Pannullo, Pisapia, Liechty, Magge, Ramakrishna. Critically revising the article: Ivanidze, Chidambaram, Pannullo, Roytman, Pisapia, Liechty, Magge, Ramakrishna. Reviewed submitted version of manuscript: all authors. Statistical analysis: Ivanidze, Chidambaram, Liechty. Administrative/technical/material support: Pisapia, Liechty. Study supervision: Ivanidze, Chidambaram, Pannullo.

\section{Correspondence}

Jana Ivanidze: Weill Cornell Medicine, New York, NY. jai9018@ med.cornell.edu. 